\title{
Solutions of elliptic equations with indefinite nonlinearities via Morse theory and linking
}

\author{
by \\ Stanley ALAMA* \\ McMaster Univ., Dept. of Math. \& Stat., Hamilton Ont., L8S 4K1 Canada \\ alamas@icarus.math.mcmaster.ca \\ and \\ Manuel DEL PINO ${ }^{\dagger}$ \\ Univ. of Chicago, Dept. of Math., Chicago IL 60637 \\ delpino@math.uchicago.edu
}

ABSTRACT. - We consider the Dirichlet problem for the equation $-\Delta u=\lambda u+h(x) f(u)$, with $h$ changing sign. In particular, we study existence of nontrivial solutions in the case where $f$ has superlinear growth, but is not assumed to be odd. Two different approaches are used: one involving Morse theory and one using min-max methods.

RÉSUMÉ. - Nous étudions le problème de Dirichlet pour l'équation $-\Delta u=\lambda u+h(x) f(u)$, où $h$ est une fonction qui change de signe. En particulier, nous établissons l'existence de solutions non triviales quand $f$ est surlinéaire, mais pas nécessairement impair. Nous nous servons de deux approches différentes, l'une basée sur la théorie de Morse, et l'autre sur les méthodes d'enlacement.

* Supported by an NSERC (Canada) Research Grant.

$\dagger$ Supported in part by NSF grant DMS 9200098 and by EC grant CI $1 *$ CT93-0323 CCE. 


\section{INTRODUCTION}

In this paper we seek nontrivial solutions for:

$$
\left\{\begin{array}{c}
-\Delta u=\lambda u+h(x) f(u) \\
\left.u\right|_{\partial \Omega}=0
\end{array}\right.
$$

where $\Omega \subset \mathbf{R}^{N}$ is a bounded open set with smooth boundary, $h \in C^{\alpha}(\bar{\Omega})$ changes sign in $\Omega$, and $f$ is a continuous function which satisfies certain superlinear, subcritical growth conditions. We mainly focus on the case $\lambda \geq \lambda_{1}(\Omega)$, where $\lambda_{1}(\Omega)$ denotes the smallest Dirichlet eigenvalue of $-\Delta$ in $\Omega$.

The existence of positive solutions to $(1.1)_{\lambda}$ with indefinite $h$ has already been established in various contexts. If the domain $\Omega$ is replaced by a compact manifold of dimension $N \geq 3$, the critical exponent case, $f(u)=u^{\frac{N+2}{N-2}}$, arises in the prescribed scalar curvature problem (see Kazdan \& Warner [14]). For manifolds carrying scalar-flat metrics, sufficient conditions for the existence of positive solutions were given by Escobar \& Schoen [12]. Ouyang [16] studied $(1.1)_{\lambda}$ on a compact manifold with homogeneous nonlinearities $f(u)=|u|^{p-2} u, p>2$, via bifurcation analysis. Results for more general subcritical $f$ were obtained by Alama \& Tarantello [1] and by Berestycki, Capuzzo-Dolcetta \& Nirenberg ([5], [7]). For instance, it is proven in [1] that if $f(u) \sim|u|^{q-2} u$ near zero, and

$$
\int_{\Omega} h(x) e_{1}^{q} d x<0
$$

(where $e_{1}$ denotes the eigenfunction corresponding to $\lambda_{1}(\Omega)$ ), then there is a finite value $\Lambda>\lambda_{1}(\Omega)$ such that equation $(1.1)_{\lambda}$ admits a positive solution for $\lambda \in\left(\lambda_{1}(\Omega), \Lambda\right)$, but $(1.1)_{\lambda}$ has no positive solutions for any $\lambda>\Lambda$. If in addition $f$ satisfies the estimate (1.7) given below, then $(1.1)_{\lambda}$ admits a second positive solution for $\lambda \in\left(\lambda_{1}(\Omega), \Lambda\right)$. In fact, condition (1.2) is an essential assumption when finding positive solutions of $(1.1)_{\lambda}$ with $\lambda \geq \lambda_{1}(\Omega)$, in the sense that it is necessary for their existence in case $f(u)=|u|^{p-2} u$. (See also Berestycki, Capuzzo-Dolcetta \& Nirenberg [6], and Tehrani [19] for related results.) Without the sign condition (1.2) but assuming that $f$ is odd, Alama \& Tarantello [1] also prove the existence of infinitely many nontrivial solutions for (nearly) every $\lambda \in \mathbb{R}$.

Our objective is to find nontrivial (possibly changing-sign) solutions of $(1.1)_{\lambda}$, without imposing either a symmetry assumption on $f$ or a sign condition on $h$ (such as (1.2)). We conjecture that $(1.1)_{\lambda}$ admits a nontrivial solution for all $\lambda \in \mathbb{R}$, assuming only superlinear growth at zero and power 
growth at infinity (see (1.3) and (1.7) introduced below). In this paper we provide some progress in this direction. We introduce the usual action functional,

$$
J_{\lambda}(u)=\int_{\Omega} \frac{1}{2}\left(|\nabla u|^{2}-\lambda u^{2}\right)-h(x) F(u) d x
$$

where $F(u)=\int_{0}^{u} f(t) d t$, and seek nontrivial critical points on $H_{0}^{1}(\Omega)$.

The main difficulty which arises in this problem lies in devising a minmax critical value for $J_{\lambda}$ when $\lambda \geq \lambda_{1}(\Omega)$. Typically, one seeks a manifold whose boundary $\Gamma$ links with a subset of $\left\{J_{\lambda} \geq \varepsilon>0\right\}$, and for which $\sup _{\Gamma} J_{\lambda}<\varepsilon$. Then a positive critical value may be defined by a min-max over all surfaces having common boundary $\Gamma$. When $h(x) \geq 0$ these sets are easily constructed, using linear subspaces of eigenfunctions of the Laplacian, and (when $f$ has subcritical growth) the equation admits a nontrivial solution for any $\lambda \in \mathbb{R}$ (cf. Rabinowitz [17]). If $h(x) \leq 0$ the results are quite different, but existence (and, in this case, nonexistence) theorems can likewise be derived from a judicious choice of linear subspaces (see [11] and [2]). When $h$ changes sign both the quadratic and superquadratic terms of $J_{\lambda}$ are indefinite, and it is not clear whether there exist linear subspaces on which both terms have the correct sign. The special case of odd $f$ is simpler since it is not necessary to construct these linking sets explicitly. This is because the Krasnoselskii genus provides for a stronger intersection property of symmetric sets, based on the Borsuk-Ulam Theorem (see [17], [1]).

A second difficulty created by the indefiniteness of $h$ is in verifying the Palais-Smale condition for the functional $J_{\lambda}$. As has already been remarked in [1], when $h$ changes sign familiar inequality conditions relating $F$ and $f=F^{\prime}$ are not helpful in deriving estimates on Palais-Smale sequences. In this paper, we follow [1] in imposing sufficient conditions on $h$ or $f$ in order to ensure that the (PS) condition holds for $I_{\lambda}$. (See Proposition 2.6.)

In order to deal with the first difficulty we use topological arguments in the spirit of earlier work by Hofer [13], Z. Q. Wang [20], and K. C. Chang [9]. Before stating our first two results, we introduce the following hypotheses and definitions: $f$ satisfies

$$
\lim _{u \rightarrow 0} \frac{f(u)}{u}=0
$$

and for some $p$ with $2<p<2^{*}$,

$$
f(u)-|u|^{p-2} u=o(|u|) \quad \text { as }|u| \rightarrow \infty .
$$

Vol. 13, $\mathrm{n}^{\circ}$ 1-1996. 
In addition, we suppose that $h$ has a "thick" zero-set,

$$
\overline{\{x: h(x)>0\}} \cap \overline{\{x: h(x)<0\}}=\emptyset .
$$

Finally, as in Ouyang [16] we define

$$
\lambda_{*}=\inf \left\{\|\nabla v\|_{2}^{2}:\|v\|_{2}=1, \int_{\Omega} h(x)|v|^{p} d x=0\right\} .
$$

Remark 1.1. - Straightforward arguments show that:

(a) $\lambda_{*} \geq \lambda_{1}(\Omega)$, with equality if and only if $\int_{\Omega} h(x) e_{1}^{p} d x=0$.

(b) $\lambda_{*}<\lambda_{1}\left(\Omega_{0}\right)$, where we denote by

$$
\Omega_{0}=\{x \in \Omega: h(x)=0\},
$$

and by $\lambda_{1}\left(\Omega_{0}\right)$ the first Dirichlet eigenvalue of $-\Delta$ in $\Omega_{0}$.

(c) One may find functions $h$ for which $\lambda_{*}$ is arbitrarily large. We provide an example to illustrate this fact in the Appendix (Section 4).

Under the above hypotheses we prove the following two existence and multiplicity results:

THEOREM 1.2. - Assume (1.3), (1.4), and (1.5) hold, $h \in C^{\alpha}(\bar{\Omega})$ changes sign in $\Omega, \lambda<\lambda_{*}$, and $\lambda$ is not a Dirichlet eigenvalue of $-\Delta$ in $\Omega$. Then $(1.1)_{\lambda}$ admits a nontrivial solution.

Combining the topological information provided in the proof of Theorem 1.2 with previous results of Wang [20] and Alama \& Tarantello [1], we may obtain some multiplicity results for $(1.1)_{\lambda}$ :

THEOREM 1.3. - Assume (1.3), (1.4), and (1.5) hold, and $h \in C^{\alpha}(\bar{\Omega})$ changes sign in $\Omega$.

i. If $\lambda<\lambda_{1}(\Omega)$, then $(1.1)_{\lambda}$ admits at least three nontrivial solutions.

ii. If

$$
\lim _{u \rightarrow 0} \frac{f(u)}{|u|^{q-2} u}=\alpha>0
$$

for some $q>2$, and (1.2) holds, then there exists $\bar{\lambda}>\lambda_{1}(\Omega)$ such that $(1.1)_{\lambda}$ admits at least five nontrivial solutions for $\lambda \in\left(\lambda_{1}(\Omega), \bar{\lambda}\right)$.

Remark 1.4. - Note that when $\lambda<\lambda_{1}(\Omega)$ the functional $J_{\lambda}$ exhibits a mountain-pass structure, and hence the first two solutions (one positive and one negative) found in part (a) of Theorem 1.3 may be obtained under the less stringent conditions of Proposition 2.6. Likewise, four of the solutions 
(two positive and two negative) claimed in part (b) of Theorem 1.3 may be derived via Theorem 2.7 of [1] under slightly weaker hypotheses.

Under the assumptions above, when $\lambda<\lambda_{*}$ we may explicitly compute the topology of negative sublevel sets of $J_{\lambda}$ and argue indirectly using the Morse inequalities. The hypotheses (1.4) and (1.5) play a central but technical role in constructing a homotopy equivalence between these sublevel sets and an infinite dimensional sphere. Indeed, condition (1.5) was also introduced in a technical capacity in [1] (also [3]) in verifying the Palais-Smale condition for variational problems associated to indefinite semilinear problems. (See Proposition 2.6.) Rather different conditions on the zero-set of $h$ were employed by Berestycki, Capuzzo-Dolcetta \& Nirenberg [5] in studying positive solutions to $(1.1)_{\lambda}$. There they consider general $f$ (satisfying (1.6) and (1.2)), but replace condition (1.5) with non-degeneracy conditions such as $\nabla h \neq 0$ on $\Omega_{0}$. The methods of [5] are based on a priori estimates and fixed point arguments, and hence differ considerably from our variational approach to the theorems stated above. Indeed, the methods of [6] can not be expected to apply directly in our setting, since the multiplicity result of [1, Theorem 3.1] (in the symmetric case $f(-u)=-f(u)$ ) demonstrates that (changing-sign) solutions of $(1.1)_{\lambda}$ are not in general a priori bounded.

Denote by $\lambda_{2}(\Omega)$ the second (min-max) eigenvalue of $-\Delta$ in $H_{0}^{1}(\Omega)$. In Section 3 we present a different approach, based on linking, to treat $(1.1)_{\lambda}$ when $\lambda_{1}(\Omega) \leq \lambda<\lambda_{2}(\Omega)$. This approach has the advantage that it demands fewer hypotheses on $f$ and $h$, although it may well be that $\lambda_{*}>\lambda_{2}(\Omega)$ for certain functions $h$. We prove:

THEOREM 1.5. - Suppose (1.3) holds,

$$
f(u)-|u|^{p-2} u=O(|u|) \quad \text { as }|u| \rightarrow \infty,
$$

for some $2<p<2^{*}$, and $h \in C^{\alpha}(\bar{\Omega})$ changes sign in $\Omega$. If $\lambda_{1}(\Omega) \leq \lambda<$ $\lambda_{2}(\Omega)$ and $\lambda$ is not a Dirichlet eigenvalue of $-\Delta$ in $\Omega_{0}$, then $(1.1)_{\lambda}$ admits a nontrivial solution.

The requirement that $\lambda$ not be a Dirichlet eigenvalue of $-\Delta$ in $\Omega_{0}$ is related to the Palais-Smale condition (see Proposition 2.6).

Finally, we note that the each of the above results continues to hold if $-\Delta$ is replaced by any symmetric, uniformly elliptic operator with self-adjoint boundary condition, or if $(1.1)_{\lambda}$ is posed on a compact manifold. 


\section{THE CASE $\lambda<\lambda_{*}$}

We define the Dirichlet Laplacian on any measurable subset $\omega \subset \Omega$ as the unique self-adjoint operator associated to the quadratic form $a(u)=\int_{\Omega}|\nabla u|^{2} d x$ with form domain

$$
H_{D}^{1}(\omega)=\left\{u \in H_{0}^{1}(\Omega): u(x)=0 \text { for a.e. } x \in \Omega \backslash \omega\right\} .
$$

When $\partial \omega$ is smooth $H_{D}^{1}(\omega)$ coincides with $H_{0}^{1}(\omega)$, and we obtain the classical Laplace operator with Dirichlet condition on $\partial \omega$. Throughout the paper we will denote the (Dirichlet) spectrum of $-\Delta$ on $\omega$ as $\sigma(\omega)=\left\{\lambda_{i}(\omega) ; i=1,2, \ldots\right\}$. Also, the letter $c$ will be indiscriminately used to denote various constants whose exact value is irrelevant.

We collect here the hypotheses for Theorem 1.2 and Theorem 1.3. First, $f$ is superlinear at zero, i.e., $f$ satisfies (1.3). At infinity, we require $f$ to be asymptotically homogeneous in the sense of (1.4) : for some $2<p<2 *$ we have,

$$
\left\{\begin{array}{c}
\text { for all } \varepsilon>0 \text { there exists a constant } C=C(\varepsilon) \text { so that } \\
\qquad\left.|f(u)-| u\right|^{p-2} u|\leq \varepsilon| u \mid+C .
\end{array}\right.
$$

(As usual, we define $2^{*}=\frac{2 N}{N-2}$ when $N \geq 3$ and $2^{*}=+\infty$ for $N=1,2$.) As consequences, we have:

$$
\begin{aligned}
F(u)-\frac{1}{p}|u|^{p} & =o\left(u^{2}\right) \\
F(u)-\frac{1}{p} f(u) u & =o\left(u^{2}\right)
\end{aligned}
$$

as $|u| \rightarrow \infty$. In addition, we assume that:

$\Omega_{+}=\{x \in \Omega: h(x)>0\} \neq \emptyset, \quad$ and $\quad \Omega_{-}=\{x \in \Omega: h(x)<0\} \neq \emptyset$, and we rewrite (1.5) as

$$
\overline{\Omega_{+}} \cap \overline{\Omega_{-}}=\emptyset
$$

The first step in proving Theorem 1.2 and Theorem 1.3 is to develop the connection between $\lambda_{*}$ and the topology of the sublevel sets of the functional $J_{\lambda}$. 
Lemma 2.1. - Assume that $f$ satisfies (2.1) and $\lambda<\lambda_{*}$. There exists a constant $K_{1}>0$ such that if $K \geq K_{1}$ and $J_{\lambda}(u) \leq-K$, then

$$
\int_{\Omega} h(x)|u|^{p} d x>0 .
$$

Proof. - We suppose the contrary: for all $n>0$ there exists $u_{n}$ with $J_{\lambda}\left(u_{n}\right) \leq-n$ and $\int_{\Omega} h(x)|u|^{p} d x \leq 0$. First note that $\left\|u_{n}\right\|_{2} \rightarrow \infty$. Indeed, $J_{\lambda}\left(u_{n}\right) \leq-n$ implies:

$$
\begin{aligned}
\frac{\lambda}{2}\left\|u_{n}\right\|_{2}^{2} & \geq n-\frac{1}{p} \int_{\Omega} h(x)\left|u_{n}\right|^{p} d x+\left[\int_{\Omega} h(x)\left(\frac{\left|u_{n}\right|^{p}}{p}-F\left(u_{n}\right)\right) d x\right] \\
& \geq n-\varepsilon\left\|u_{n}\right\|_{2}^{2}-C_{\varepsilon}
\end{aligned}
$$

using (2.3) Taking $\varepsilon$ sufficiently small, we have $\left\|u_{n}\right\|_{2} \rightarrow \infty$ as claimed.

Set $v_{n}=u_{n} /\left\|u_{n}\right\|_{2}$. We have

$$
\frac{1}{2}\left\|\nabla v_{n}\right\|_{2}^{2}-\frac{\lambda}{2}-\int_{\Omega} h(x) \frac{F\left(u_{n}\right)}{\left\|u_{n}\right\|_{2}^{2}} d x \leq 0 .
$$

Using (2.2), we see

$$
\int_{\Omega} h(x) \frac{F\left(u_{n}\right)}{\left\|u_{n}\right\|_{2}^{2}} d x=\left\|u_{n}\right\|_{2}^{p-2} \int_{\Omega} h(x)\left|v_{n}\right|^{p}+o(1) \leq o(1),
$$

since we are assuming that $\int_{\Omega} h(x)\left|u_{n}\right|^{p} d x \leq 0$. Hence, (2.5) yields

$$
\frac{1}{2}\left\|\nabla v_{n}\right\|_{2}^{2} \leq \frac{\lambda}{2}+o(1)
$$

and $v_{n} \rightarrow v_{0}$ in $H_{0}^{1}(\Omega)$ with $\left\|v_{0}\right\|_{2}=1$ and

$$
\left\|\nabla v_{0}\right\|_{2}^{2} \leq \lambda
$$

Moreover, from (2.3), (2.5) we have

$$
\begin{aligned}
0 \geq\left\|u_{n}\right\|_{2}^{p-2} \int_{\Omega} h(x)\left|v_{n}\right|^{p} d x & =\int_{\Omega} h(x) \frac{F\left(u_{n}\right)}{\left\|u_{n}\right\|_{2}^{2}} d x+o(1) \\
& \geq \frac{1}{2}\left(\left\|\nabla v_{n}\right\|_{2}^{2}-\lambda\left\|v_{n}\right\|_{2}^{2}\right)+o(1) \\
& \geq-\frac{\lambda}{2}+o(1) .
\end{aligned}
$$


Hence,

$$
\int_{\Omega} h(x)\left|v_{0}\right|^{p} d x=\lim _{n \rightarrow \infty} \int_{\Omega} h(x)\left|v_{n}\right|^{p} d x=0 .
$$

But when $\lambda<\lambda_{*},(2.6)$ contradicts the definition of $\lambda_{*}$.

We introduce the notation

$$
J_{\lambda}^{a}=\left\{v \in H_{0}^{1}(\Omega): J_{\lambda}(v) \leq a\right\} .
$$

Then, Lemma 2.1 implies that there exists a constant $K_{1}$ such that

$$
J_{\lambda}^{-K} \subset A=\left\{v \in H_{0}^{1}(\Omega): \int_{\Omega} h(x)|v|^{p} d x>0\right\}
$$

for all $K \geq K_{1}$. In fact, we will show that $J_{\lambda}^{-K}$ is a retract of $A$ for all $K$ sufficiently large.

Note that if $u \in A$, then $J_{\lambda}(t u) \rightarrow-\infty$ as $t \rightarrow \infty$. We will now show that there is a continuous choice of $T=T(u)$ for which $J_{\lambda}(T(u) u) \leq-K$ for any large $K$ and for all $u \in A$. Then we may use this construction to define our retraction.

Lemma 2.2. - Suppose $f$ satisfies (2.1) and $\lambda<\lambda_{*}$. Then there exists a constant $K_{2} \geq K_{1}$ such that whenever $J_{\lambda}(u) \leq-K_{2}$, then

$$
\left.\frac{\partial}{\partial t}\right|_{t=1} J_{\lambda}(t u)<0
$$

Proof. - From Lemma 2.1, if $K \geq K_{1}$ and $J_{\lambda}(u) \leq-K$, then $u \in A$. Suppose (to obtain a contradiction) that for every $n \geq K_{1}$ there exists $u_{n} \in A$ with $J_{\lambda}\left(u_{n}\right) \leq-n$ and

$$
\left.\frac{\partial}{\partial t}\right|_{t=1} J_{\lambda}\left(t u_{n}\right) \geq 0
$$

Then,

$$
\begin{aligned}
& \frac{1}{2}\left\|\nabla u_{n}\right\|_{2}^{2}-\frac{\lambda}{2}\left\|u_{n}\right\|_{2}^{2}-\int_{\Omega} h(x) F\left(u_{n}\right) d x \leq-n \\
& \left\|\nabla u_{n}\right\|_{2}^{2}-\lambda\left\|u_{n}\right\|_{2}^{2}-\int_{\Omega} h(x) f\left(u_{n}\right) u_{n} d x \geq 0
\end{aligned}
$$

As in Lemma 2.1, we claim that $\left\|u_{n}\right\|_{2} \rightarrow \infty$. Indeed, if along some subsequence $\left\|u_{n}\right\|_{2} \leq C$, then

$$
\begin{aligned}
& \left(\frac{1}{2}-\frac{1}{p}\right)\left(\left\|\nabla u_{n}\right\|_{2}^{2}-\lambda\left\|u_{n}\right\|_{2}^{2}\right) \\
& \leq-n+\int_{\Omega} h(x)\left[F\left(u_{n}\right)-\frac{1}{p} f\left(u_{n}\right) u_{n}\right] d x \leq C
\end{aligned}
$$


(via hypothesis (2.3)). Hence, a subsequence $u_{n} \rightarrow u_{0}$ in $H_{0}^{1}(\Omega)$, and

$$
J_{\lambda}\left(u_{0}\right) \leq \liminf _{n \rightarrow \infty} J_{\lambda}\left(u_{n}\right)=-\infty,
$$

which is impossible. Therefore $\left\|u_{n}\right\|_{2} \rightarrow \infty$.

As before, we set $v_{n}=u_{n} /\left\|u_{n}\right\|_{2}$. Then (2.7), (2.8) yield:

$$
\begin{aligned}
\left\|\nabla v_{n}\right\|_{2}^{2}-\lambda\left\|v_{n}\right\|_{2}^{2} & \leq \frac{C}{\left\|u_{n}\right\|_{2}} \int_{\Omega} h(x)\left[F\left(u_{n}\right)-\frac{1}{p} f\left(u_{n}\right) u_{n}\right] d x+o(1) \\
& \leq o(1) .
\end{aligned}
$$

Hence, $v_{n} \rightarrow v_{0}$ in $H_{0}^{1}(\Omega)$, and $\left\|\nabla v_{0}\right\|_{2}^{2} \leq \lambda$. Combining (2.7) and (2.8) in a different way we have

$$
\int_{\Omega} h(x)\left[\frac{1}{2} f\left(u_{n}\right) u_{n}-F\left(u_{n}\right)\right] d x \leq-n<0,
$$

and so (in view of (2.1) and (2.2)) we obtain:

$$
\begin{aligned}
0 & \leq\left(\frac{1}{2}-\frac{1}{p}\right)\left\|u_{n}\right\|_{2}^{p-2} \int_{\Omega} h(x)\left|v_{n}\right|^{p} d x \\
& =\int_{\Omega} h(x) \frac{\frac{1}{2} f\left(u_{n}\right) u_{n}-F\left(u_{n}\right)}{\left\|u_{n}\right\|_{2}^{2}} d x+o(1) \leq o(1) .
\end{aligned}
$$

Since $v_{n} \rightarrow v_{0}$ strongly in $L^{p}(\Omega)$, we must have

$$
\int_{\Omega} h(x)\left|v_{0}\right|^{p} d x=0,
$$

But $\left\|\nabla v_{0}\right\| \leq \lambda<\lambda_{*}$, so this contradicts the definition of $\lambda_{*}$.

We may now construct our retraction. Let $u \in A$. By Lemma 2.2, there is a unique $T=T(u)$ such that $J_{\lambda}(T u)=-K_{2}$. Moreover, by applying the Implicit Function Theorem to the map

$$
\begin{gathered}
\mathcal{F}: \mathbb{R} \times H_{0}^{1}(\Omega) \rightarrow \mathbb{R} \\
\mathcal{F}(t, u)=J_{\lambda}(t u)
\end{gathered}
$$

at $(t, u)=(T(u), u)$ it follows that $T(u)$ is a continuous function on $A$. Set $\tilde{T}(u)=\max \{T(u), 1\}$, also a continuous function on $A$. Then, define:

$$
\begin{aligned}
\eta(s, u) & =(1-s) u+s \tilde{T}(u) u \\
& =[1+s(\tilde{T}(u)-1)] u
\end{aligned}
$$


Clearly, $\eta(0, u)=u$ for all $u \in A$, and by Lemma 2.2, $\eta(1, u)=\tilde{T}(u) u \in$ $J_{\lambda}^{-K_{2}}$ for all $u \in A$. Furthermore, Lemma 2.2 and the definition of $\tilde{T}(u)$ ensure that $\eta(s, u)=u$ for all $u \in J_{\lambda}^{-K_{2}}$. In conclusion, we have shown that $\eta:[0,1] \times A \rightarrow J_{\lambda}^{-K_{2}}$ is a strong deformation retraction:

Lemma 2.3. - Suppose $f$ satisfies (2.1) and $\lambda<\lambda_{*}$. Then, $J_{\lambda}^{-K_{2}}$ is a strong retract of $A$. In particular, $J_{\lambda}^{-K_{2}}$ and $A$ are homotopically equivalent.

Now we examine the topology of the set $A$. Namely, we will show that $A$ is homotopically equivalent to an infinite dimensional sphere (and hence is contractible.)

LEMmA 2.4. - Suppose (2.4) holds. Then, $B=H_{D}^{1}\left(\Omega_{+}\right) \backslash\{0\}$ is a retract of $A$. (In particular, $A$ and $B$ are homotopically equivalent.)

Proof. - The retraction will be constructed in two steps: first we use hypothesis (2.4) to truncate $u \in A$ to have support only in $\Omega_{1}:=\{x \in \Omega: h(x) \geq 0\}$. (This is not in itself a retraction.) Then we project the resulting function linearly into $H_{D}^{1}\left(\Omega_{+}\right)$.

By (2.4), there is a function $\psi \in C^{\infty}\left(\mathbb{R}^{N}\right)$ which satisfies:

$$
\begin{cases}\psi(x)=1 & \text { for all } x \in \overline{\Omega_{+}}, \\ \psi(x)=0 & \text { for all } x \in \overline{\Omega_{-}}, \\ 0 \leq \psi(x) \leq 1 & \text { for all } x \in \Omega_{0} .\end{cases}
$$

Consider also the projection operator $P: H_{0}^{1}(\Omega) \rightarrow H_{D}^{1}\left(\Omega_{+}\right)$. Then, define

$$
\eta(s, u)= \begin{cases}(1-2 s) u+2 s \psi u, & \text { if } 0 \leq s \leq \frac{1}{2} \\ 2(1-s) \psi u+2\left(s-\frac{1}{2}\right) P(\psi u), & \text { if } \frac{1}{2} \leq s \leq 1\end{cases}
$$

Clearly, $\eta(0, u)=u$ if $u \in A$ and $\eta(s, u)=u$ for all $u \in H_{D}^{1}\left(\Omega_{+}\right)$and for all $s \in[0,1]$. It remains only to show that $\eta(s, u) \in A$ for all $s \in[0,1]$, and that $\eta(1, u) \in H_{D}^{1}\left(\Omega_{+}\right) \backslash\{0\}$.

First, note that if $u \in A$ and $0 \leq s \leq \frac{1}{2}$,

$$
\begin{aligned}
\int_{\Omega} h(x)|\eta(s, u)|^{p} d x & =\int_{\Omega_{+}} h^{+}(x)|u|^{p} d x-(1-2 s)^{p} \int_{\Omega_{-}} h^{-}(x)|u|^{p} d x \\
& \geq \int_{\Omega} h(x)|u|^{p} d x>0 .
\end{aligned}
$$

When $\frac{1}{2}<s \leq 1$, then clearly $\int_{\Omega} h(x)|\eta(s, u)|^{p} d x \geq 0$. If equality holds, then $\eta(s, u)=0$ on $\Omega_{+}$. But, in that case,

$$
P(\psi u)(x)=-\left(\frac{1-s}{s-\frac{1}{2}}\right) \psi(x) u(x) \quad \text { on } \Omega_{+} .
$$


This can only be the case if $P(\psi u)=0$, and hence (from the definition of $\psi$ in (2.9)) we have $u=0$ on $\Omega_{+}$. But this is impossible, since $u \in A$. Hence we may conclude that $\eta(s, u) \in A$ for all $s \in[0,1]$ and $u \in A$. A similar argument shows that

$$
P(\psi u)=\eta(1, u) \in H_{D}^{1}\left(\Omega_{+}\right) \backslash\{0\}
$$

Hence, $\eta$ is a retraction.

Putting Lemma 2.4 and Lemma 2.3 together, we obtain:

Proposition 2.5. - Suppose $f$ satisfies (2.1) and $\lambda<\lambda_{*}$. Let $K_{2}$ be chosen as in Lemma 2.2. Then $J_{\lambda}^{-K_{2}}$ is homotopically equivalent to an infinite dimensional sphere $S^{\infty}$.

At this point, we will use the topological information obtained in Lemmas 2.3 and 2.4 to infer the existence of nontrivial solutions of equation $(1.1)_{\lambda}$, via Morse Theory. An essential step is these arguments is the PalaisSmale condition for the functional $J_{\lambda}$. Although our hypotheses assume subcritical growth for $J_{\lambda}$ at infinity, (PS) is a nontrivial issue in this problem. We will use the following version of (PS), derived in [1].

Proposition 2.6. - Suppose $\lambda \notin \sigma\left(\Omega_{0}\right)$. Then, $J_{\lambda}$ satisfies (PS) provided either of the following two conditions hold:

$i$. For some $p, 2<p<2^{*}$, and constants $A, B>0$ we have:

$$
\left.|f(u)-| u\right|^{p-2} u|\leq A| u \mid+B .
$$

ii. Hypothesis (2.4) holds, and $f$ satisfies

$$
\lim _{|u| \rightarrow \infty} \frac{f(u)}{|u|^{p-2} u}=1, \quad \text { and } \quad\left|f^{\prime}(u)\right| \leq A|u|^{p-2}+B
$$

for some $p, 2<p<2^{*}$, with $A, B>0$ constants.

(Recall that $\sigma\left(\Omega_{0}\right)$ denotes the collection of Dirichlet eigenvalues of $-\Delta$ in $\Omega_{0}$.) For the reader's convenience, we provide a short proof of Proposition 2.6 assuming condition i. in Section 4.

Proof of Theorem 1.2. - By hypothesis (1.4) and the fact $\lambda_{*}<\lambda_{1}\left(\Omega_{0}\right)$ (see Remark 1.1) we may conclude from Proposition 2.6 that the PalaisSmale condition holds under the given assumptions. Denote by $H_{k}(X, Y)$ the $k$ th relative homology group with integer coefficients. For $v$ an isolated critical point with $J_{\lambda}(v)=c$ and $U_{v}$ a neighborhood of $v$, set

$$
C_{k}\left(J_{\lambda}, v\right)=H_{k}\left(J_{\lambda}^{c} \cap U_{v},\left[J_{\lambda}^{c} \cap U_{v}\right] \backslash\{v\}\right), \quad k=0,1, \ldots,
$$


the $k$ th critical group at the critical point $v$. If $J_{\lambda}$ has only finitely many critical points $u_{1}, \ldots, u_{n}$ with $a<J_{\lambda}\left(u_{j}\right)<b$, we define the Morse numbers of the pair $\left(J_{\lambda}^{b}, J_{\lambda}^{a}\right)$ by

$$
M_{k}=M_{k}\left(J_{\lambda}^{b}, J_{\lambda}^{a}\right)=\sum_{j=1}^{n} \operatorname{dim} C_{k}\left(J_{\lambda}, u_{j}\right) .
$$

Then, if $\beta_{k}=\operatorname{dim} H_{k}\left(J_{\lambda}^{b}, J_{\lambda}^{a}\right)$ are the Betti numbers of the pair $\left(J_{\lambda}^{b}, J_{\lambda}^{a}\right)$, the Morse inequalities require that:

$$
\sum_{k=0}^{\infty}(-1)^{k} M_{k}=\sum_{k=0}^{\infty}(-1)^{k} \beta_{k}
$$

(For a derivation of (2.11) and other facts from infinite-dimensional Morse Theory see Mawhin \& Willem [15] or Chang [9].)

To prove Theorem 1.2, we argue by contradiction and assume that 0 is the only critical point of $J_{\lambda}$. By hypothesis $\lambda \notin \sigma(\Omega)$, and hence the Morse numbers of the pair $\left(H_{0}^{1}(\Omega), J_{\lambda}^{-K_{2}}\right)$ are

$$
M_{k}=\operatorname{dim} C_{k}\left(J_{\lambda}, 0\right)=\delta_{k, m},
$$

where $m$ is the Morse index of the (non-degenerate) critical point 0 . On the other hand, from Proposition 2.5 and Proposition 2.6, $J_{\lambda}^{-K_{2}}$ is contractible in $H_{0}^{1}(\Omega)$. In particular,

$$
H_{k}\left(H_{0}^{1}(\Omega), J_{\lambda}^{-K_{2}}\right)=H_{k}\left(H_{0}^{1}(\Omega), S^{\infty}\right)=\{0\}, \quad \text { for all } k=0,1,2, \ldots
$$

and so the Betti numbers of the pair $\left(H_{0}^{1}(\Omega), J_{\lambda}^{-K_{2}}\right)$ all vanish, $\beta_{k}=0$, $k=1,2, \ldots$. But this contradicts the Morse inequality (2.11), and hence $J_{\lambda}$ must admit a nontrivial critical point.

Remark 2.7. - Extending the proof of Theorem 1.2 to include the case where $\lambda$ is an eigenvalue of $-\Delta$ in $\Omega$ depends on obtaining some additional information about the critical groups $C_{k}\left(J_{\lambda}, 0\right)$. For example, if $f$ satisfies a estimate such as (1.6) we may determine $C_{k}\left(J_{\lambda_{1}}, 0\right)$ and obtain a solution at $\lambda=\lambda_{1}(\Omega)$ when $\int_{\Omega} h(x) e_{1}^{q} d x$ is nonzero. In general, however, one cannot expect that critical points with Morse index $m \geq 1$ have nontrivial critical groups: consider $\varphi(x, y)=y^{3}-x^{2}$ for which all critical groups are trivial.

Remark 2.8. - Theorem 1.2 may also be proven via min-max arguments. If $\lambda<\lambda_{1}(\Omega)$ we may obtain two solutions by a straightforward application of the Mountain Pass Theorem (see Remark 1.1), so we assume that the 
Morse index of 0 is $m \geq 1$. Since 0 is a nondegenerate critical point, we can decompose $H_{0}^{1}(\Omega)=X^{-} \oplus X^{+}$by the eigenspaces of $-\Delta$, with $\operatorname{dim} X^{-}=m$, and

$$
\sup _{X-\cap S_{\delta}} J_{\lambda} \leq-\varepsilon<0 \text { and } \inf _{X+\cap S_{\delta}} J_{\lambda} \geq+\varepsilon>0
$$

for some choice of $\varepsilon, \delta>0$. Now, if $J_{\lambda}$ has no critical points other than zero, there exists a deformation (obtained by a negative pseudogradient flow) $\eta:[0,1] \times H_{0}^{1}(\Omega) \rightarrow H_{0}^{1}(\Omega)$, such that $\eta(0, v)=v, J_{\lambda}(\eta(1, v))<-K_{2}$ for all $v \in S_{\delta} \cap X^{-}, \eta(t, \cdot)$ is a homeomorphism for each $t$, and $J_{\lambda}(\eta(t, v)) \leq-\varepsilon<0$ for all $(t, v) \in[0,1] \times\left(S_{\delta} \cap X^{-}\right)$. Moreover, note that $\left\{\eta(t, v): t \in[0,1], v \in S_{\delta} \cap X^{-}\right\}$defines a smooth manifold of dimension $m$ in $H_{0}^{1}(\Omega)$. Define a surface $\Sigma_{1}$ by:

$$
\Sigma_{1}=\left[B_{\delta} \cap X^{-}\right] \cup\left\{\eta(t, v): t \in[0,1], v \in S_{\delta} \cap X^{-}\right\} .
$$

By Proposition 2.5, $\partial \Sigma_{1}=\left\{\eta(1, v): v \in S_{\delta} \cap X^{-}\right\}$is contractible in $J_{\lambda}^{-K_{2}}$, so we may "close" $\Sigma_{1}$ to form a surface $\Sigma$ with $\sup _{\Sigma} J_{\lambda} \leq 0$, and which links the sphere $S_{\delta} \cap X^{+}$. The linking theorem of Benci \& Rabinowitz [4] would then give a nontrivial critical point of $J_{\lambda}$. The details are left to the interested reader.

Proof of Theorem 1.3. - To prove part $i$. we follow Wang [20] (see also Theorem III.2.3 of Chang [9].) When $\lambda<\lambda_{1}(\Omega)<\lambda_{1}\left(\Omega_{0}\right)$ Proposition 2.6 applies and hence the Palais-Smale condition is satisfied by $J_{\lambda}$. Moreover, when $\lambda<\lambda_{1}(\Omega)$ and $p<\frac{2 N}{N-2}$, zero is a strict local minimum for $J_{\lambda}$, so $m_{k}(0):=\operatorname{dim} C_{k}\left(J_{\lambda}, 0\right)=\delta_{0, k}$.

The first two nontrivial solutions will be obtained via the Mountain-pass Theorem. Define

$$
J_{ \pm}(u)=\frac{1}{2} \int_{\Omega}|\nabla u|^{2}-\lambda\left(u_{ \pm}\right)^{2}-\int_{\Omega} h(x) F\left(u_{ \pm}\right) d x,
$$

where $u_{+}=\max \{u(x), 0\} \geq 0, u_{-}=\min \{u(x), 0\} \leq 0$. Clearly there exists $v_{0} \geq 0$ such that $J_{ \pm}\left( \pm v_{0}\right)=J_{\lambda}\left( \pm v_{0}\right)<0$. Since $\lambda<\lambda_{1}(\Omega)<$ $\lambda_{1}\left(\Omega_{0}\right)$, (PS) holds for $J_{\lambda}$, and also for $J_{ \pm}$. Applying familiar arguments we obtain critical points $u_{+}>0$ of $J_{+}$and $u_{-}<0$ for $J_{-}$, which are nontrivial solutions of $(1.1)_{\lambda}$ and critical points for $J_{\lambda}$.

To obtain the third solution, we must determine the critical groups of $u_{ \pm}$. By Corollary 8.5 in [15], if these are the only nontrivial solutions of $(1.1)_{\lambda}$ we have:

$$
\operatorname{dim} C_{k}\left(J_{ \pm}, u_{ \pm}\right)=\delta_{1 k}
$$


Denote by $\tilde{J}, \tilde{J}_{ \pm}$the restrictions of $J_{\lambda}$ and $J_{ \pm}$(respectively) to $C_{0}^{1}(\bar{\Omega})$. By Theorem 1 in [10] (see also Corollary III.1.2 in [9]), the critical groups of $u_{ \pm}$are the same in $H_{0}^{1}(\Omega)$ as in the dense subspace $C_{0}^{1}(\bar{\Omega})$ :

$$
C_{k}\left(J_{\lambda}, u_{ \pm}\right)=C_{k}\left(\tilde{J}, u_{ \pm}\right) \quad \text { and } \quad C_{k}\left(J_{ \pm}, u_{ \pm}\right)=C_{k}\left(\tilde{J}_{ \pm}, u_{ \pm}\right) .
$$

On the other hand, $\tilde{J}=\tilde{J}_{ \pm}$when restricted to a small $C_{0}^{1}$ neighborhood of $u_{ \pm}$, so $C_{k}\left(\tilde{J}, u_{ \pm}\right)=C_{k}\left(\tilde{J}_{ \pm}, u_{ \pm}\right)$, and hence

$$
m_{k}\left(u_{ \pm}\right):=\operatorname{dim} C_{k}\left(J_{\lambda}, u_{ \pm}\right)=\delta_{1 k} .
$$

Now, if $0, u_{ \pm}$were the only critical points of $J_{\lambda}$, we would have

$$
M_{k}=m_{k}(0)+m_{k}\left(u_{+}\right)+m_{k}\left(u_{-}\right)= \begin{cases}1, & \text { if } k=0 \\ 2, & \text { if } k=1, \\ 0, & \text { if } k \geq 2\end{cases}
$$

Since $\lambda<\lambda_{1}(\Omega) \leq \lambda_{*}$, by Proposition 2.5 we again have Betti numbers $\beta_{k}=0, k=0,1,2, \ldots$, and the Morse inequality (2.11) is violated. This proves $i$.

Finally, we prove $i i$. Assume that (1.6) and (1.2) hold, and $\lambda>\lambda_{1}(\Omega)$. In [1] it is proven that there exists $\Lambda_{+}>\lambda_{1}(\Omega)$ such that $(1.1)_{\lambda}$ admits a pair of positive solutions for each $\lambda \in\left(\lambda_{1}(\Omega), \Lambda_{+}\right)$. By the exact same arguments there exists $\Lambda_{-}>\lambda_{1}(\Omega)$ such that $(1.1)_{\lambda}$ admits a pair of negative solutions for each $\lambda \in\left(\lambda_{1}(\Omega), \Lambda_{-}\right)$. In order to obtain the fifth nontrivial solution we must determine the critical groups for each of these four solutions, and so we briefly review their derivation in [1].

Let $\bar{\lambda}=\min \left\{\lambda_{*}, \Lambda_{+}, \Lambda_{-}, \lambda_{2}(\Omega)\right\}$. Recall from Remark 1.1 that hypothesis (1.2) ensures that $\lambda_{*}>\lambda_{1}(\Omega)$, and hence $\bar{\lambda}>\lambda_{1}(\Omega)$. Moreover we have $\bar{\lambda} \leq \lambda_{*}<\lambda_{1}\left(\Omega_{0}\right)$, so (PS) holds for $J_{\lambda}$ when $\lambda<\bar{\lambda}$. Furthermore, if $\lambda \in\left(\lambda_{1}(\Omega), \bar{\lambda}\right)$, we have

$$
m_{k}(0)=\operatorname{dim} C_{k}\left(J_{\lambda}, 0\right)=\delta_{1 k} .
$$

It is easy to see that for all $\lambda>\lambda_{1}(\Omega)$ there exists $t_{0}=t_{0}(\lambda)>0$ such that for $0<t \leq t_{0}, \underline{v}=t e_{1}$ is a subsolution and $\bar{w}=-t e_{1}$ is a supersolution of $(1.1)_{\lambda}$. In addition, a positive solution $\bar{v}$ of $(1.1)_{\mu}$ with $\mu>\lambda$ is a supersolution for $(1.1)_{\lambda}$, while a negative solution $\underline{w}$ of $(1.1)_{\mu}$ with $\mu>\lambda$ is a subsolution for $(1.1)_{\lambda}$. By choosing $t>0$ sufficiently small so that $0<\underline{v} \leq \bar{v}$ and $\underline{w} \leq \bar{w}<0$ we may determine a positive solution $u_{\lambda}^{+}>0$ and a negative solution $u_{\lambda}^{-}<0$ to $(1.1)_{\lambda}$ via minimization,

$$
\begin{aligned}
& J_{\lambda}\left(u_{\lambda}^{+}\right)=\inf \left\{J_{\lambda}(u): 0<\underline{v} \leq u \leq \bar{v}\right\} \\
& J_{\lambda}\left(u_{\lambda}^{-}\right)=\inf \left\{J_{\lambda}(u): \underline{w} \leq u \leq \bar{w}<0\right\} .
\end{aligned}
$$


(See [18].) Since $u_{\lambda}^{ \pm}$represent minimizers of $J_{\lambda}$ in the $C_{0}^{1}(\bar{\Omega})$ topology, by a result of Brezis \& Nirenberg [8] they are also minimizers in $H_{0}^{1}(\Omega)$. We assume that both $u_{\lambda}^{ \pm}$are isolated minima, (otherwise, we obtain infinitely many solutions), and hence

$$
m_{k}\left(u_{\lambda}^{ \pm}\right)=\operatorname{dim} C_{k}\left(J_{\lambda}, u_{\lambda}^{ \pm}\right)=\delta_{0 k} .
$$

(See also [10].)

To obtain the second pair of single-sign solutions, we appeal to the Mountain-pass Theorem. Define functionals

$$
\begin{aligned}
I_{ \pm}(v)= & \frac{1}{2} \int_{\Omega}|\nabla v|^{2} \\
& -\lambda\left(v_{ \pm}\right)^{2}-\int_{\Omega} h(x)\left[F\left(u_{\lambda}^{ \pm}+v_{ \pm}\right)-F\left(u_{\lambda}^{ \pm}\right)-f\left(u_{\lambda}^{ \pm}\right) v_{ \pm}\right] d x
\end{aligned}
$$

where (as before) $v_{+}=\max \{v(x), 0\} \geq 0, v_{-}=\min \{v(x), 0\} \leq 0$. Note that if $v \geq 0$ or $v \leq 0$, then $I_{ \pm}(v)=J_{\lambda}\left(u_{\lambda}^{ \pm}+v\right)-J_{\lambda}\left(u_{\lambda}^{ \pm}\right)$. Simple calculations show that: $v=0$ is a strict local minimum for $I_{ \pm}$; there exists $v_{0} \geq 0$ such that $I_{ \pm}\left( \pm t v_{0}\right) \rightarrow-\infty$ as $t \rightarrow+\infty$; and $I_{ \pm}$ satisfy the Palais-Smale condition. (See Section 2 of [1] for the detailed computations.) Applying the Mountain-pass Theorem to $I_{ \pm}$we obtain nontrivial critical points $v^{+} \geq 0, v^{-} \leq 0$ which give rise to solutions of $(1.1)_{\lambda}$, (and hence critical points of $J_{\lambda}$, ) $w_{\lambda}^{+}=u_{\lambda}^{+}+v^{+}>u_{\lambda}^{+}>0$ and $w_{\lambda}^{-}=u_{\lambda}^{-}+v^{-}<u_{\lambda}^{-}<0$.

Now we repeat the analysis of part $i$. to calculate the corresponding critical groups. Restricted to a $C_{0}^{1}(\bar{\Omega})$ neighborhood of $v_{\lambda}^{ \pm}$, we have $I_{ \pm}(v)=J_{\lambda}\left(u_{\lambda}^{ \pm}+v\right)-J_{\lambda}\left(u_{\lambda}^{ \pm}\right)$. Therefore we may apply Corollary 8.5 of [15] and Theorem 1 of [10] to obtain

$$
m_{k}\left(w_{\lambda}^{ \pm}\right):=\operatorname{dim} C_{k}\left(J_{\lambda}, w_{\lambda}^{ \pm}\right)=\operatorname{dim} C_{k}\left(I_{ \pm}, v^{ \pm}\right)=\delta_{1 k} .
$$

Hence, if $0, u_{\lambda}^{ \pm}, w_{\lambda}^{ \pm}$were the only solutions of $(1.1)_{\lambda}$, we would have Morse numbers

$$
\begin{aligned}
M_{k} & =m_{k}(0)+m_{k}\left(u_{\lambda}^{+}\right)+m_{k}\left(u_{\lambda}^{-}\right)+m_{k}\left(w_{\lambda}^{+}\right)+m_{k}\left(w_{\lambda}^{-}\right) \\
& = \begin{cases}2, & \text { if } k=0, \\
3, & \text { if } k=1, \\
0, & \text { if } k \geq 2 .\end{cases}
\end{aligned}
$$

Again, this contradicts the Morse inequality (2.11) over $\left(H_{0}^{1}(\Omega), J_{\lambda}^{-K_{2}}\right)$, and so there must exist another nontrivial solution. 


\section{A LINKING APPROACH}

We prove the following, which includes Theorem 1.5:

THEOREM 3.1. - Suppose $f$ satisfies the hypotheses of Proposition 2.6 and $\lambda_{1}(\Omega) \leq \lambda<\lambda_{2}(\Omega)$. Then (1.1) ${ }_{\lambda}$ possesses at least one nontrivial solution.

Define the linear subspace $V=\left(\operatorname{span}\left\langle e_{1}\right\rangle\right)^{\perp}$, and let $w_{1}, w_{2} \in C_{0}^{\infty}\left(\Omega_{+}\right)$ be fixed functions with supp $w_{1}$, supp $w_{2}$ disjoint. Choose $R>0$ sufficiently large so that

$$
J_{\lambda}\left( \pm R w_{1}\right)<0 \quad \text { and } \quad J_{\lambda}\left( \pm R w_{1}\right)<-\max _{t \in[0, \infty)} J_{\lambda}\left(t w_{2}\right)
$$

Since $f$ has superlinear growth at 0 , there exists a radius $\rho>0$ and constant $\alpha>0$ with

$$
J(u) \geq \alpha>0 \text { for } u \in V \cap S_{\rho} .
$$

Introduce the modified functionals

$$
J_{ \pm}(u)=\int_{\Omega} \frac{1}{2}\left[|\nabla u|^{2}-\lambda\left(u_{ \pm}\right)^{2}\right]-h(x) F\left(u_{ \pm}\right) d x .
$$

Standard arguments show that a critical point of $J_{ \pm}$corresponds to a positive (respectively, negative) solution of $(1.1)_{\lambda}$. Set

$$
\begin{aligned}
& \beta_{ \pm}=\inf _{\gamma \in \Gamma_{ \pm}} \sup _{t \in[0,1]} J_{ \pm}(\gamma(t)) \\
& \Gamma_{ \pm}=\left\{\gamma \in C\left([0,1] ; H_{0}^{1}(\Omega)\right): \gamma(0)=0, \gamma(1)= \pm R w_{1}\right\} .
\end{aligned}
$$

Clearly, $\beta_{ \pm} \geq 0$. If either $\beta_{ \pm}>0$, then we obtain a positive (respectively, negative) solution to $(1.1)_{\lambda}$ with critical value $\beta_{ \pm}>0$, and hence a nontrivial solution to $(1.1)_{\lambda}$.

Suppose that both $\beta_{ \pm}=0$. Then there exist curves $\gamma_{ \pm} \in \Gamma_{ \pm}$with

$$
J_{ \pm}\left(\gamma_{ \pm}(t)\right) \leq \frac{\alpha}{2}
$$

Without loss, we may suppose that $\gamma_{ \pm}$are simple curves, and that $\gamma_{+}(t) \geq 0$, $\gamma_{-}(t) \leq 0$ for all $t \in[0,1]$, with $\gamma_{ \pm}(t) \equiv 0$ if and only if $t=0$. Since $V$ is characterized by

$$
v \in V \quad \text { if and only if } \int_{\Omega} v e_{1}=0,
$$

we have $\gamma_{ \pm}(t) \in B_{\rho} \cap V$ if and only if $t=0$. 
Finally, we connect $R w_{1}$ to $-R w_{1}$ by a path $\gamma_{0} \subset H_{D}^{1}\left(\Omega_{+}\right)$with

$$
J_{\lambda}\left(\gamma_{0}(\cdot)\right)<0 \text { and }\left\|\gamma_{0}\right\|_{H_{D}^{1}\left(\Omega_{+}\right)}>\rho .
$$

To do this, choose $T>0$ such that

$$
J_{\lambda}\left(T w_{2}\right)<0 \quad \text { and } \quad J_{\lambda}\left(T w_{2}\right)<-\max _{t \in[-R, R]} J_{\lambda}\left(t w_{1}\right)
$$

Then, let

$$
\gamma_{0}(t)= \begin{cases}w+3 t T w_{2}, & \text { when } t \in\left[0, \frac{1}{3}\right] \\ T w_{2}+3(1-2 t) w, & \text { when } t \in\left[\frac{1}{3}, \frac{2}{3}\right] \\ -w+(3-3 t) T w_{2}, & \text { when } t \in\left[\frac{1}{2}, 1\right]\end{cases}
$$

Since $w_{1}, w_{2}$ have disjoint supports, $J_{\lambda}\left(\alpha w+\beta w_{2}\right)=J_{\lambda}(\alpha w)+J_{\lambda}\left(\beta w_{2}\right)$, and the choice of $R$ and $T$ ensure that $J_{\lambda}\left(\gamma_{0}(t)\right)<0$ for all $t \in[0,1]$.

Let $C$ be the closed curve obtained by joining $\gamma_{+}$to $\gamma_{0}$ to $\gamma_{-}$. By construction, $C$ intersects $S_{\rho} \cap V$ only at 0 . Parametrize $C$ by a one-to-one map $\psi: S^{1} \rightarrow C \subset H_{0}^{1}(\Omega)$ with $\psi\left(e^{i 0}\right)=0$, and set

$$
\Sigma=\left\{\sigma \in C\left(D^{2} ; H_{0}^{1}(\Omega)\right):\left.\sigma\right|_{S^{1}}=\psi\right\}
$$

(where we write $D^{2}$ to represent the unit disk in $\mathbb{R}^{2}$ with boundary $\left.\partial D^{2}=S^{1}\right)$. Then, we have

$$
\left.J_{\lambda}\right|_{C} \leq \frac{\alpha}{2} \quad \text { and }\left.\quad J_{\lambda}\right|_{S_{\rho} \cap V} \geq \alpha .
$$

Let

$$
b=\inf _{\sigma \in \Sigma} \sup _{\xi \in D^{2}} J_{\lambda}(\sigma(\xi)) .
$$

If we can show that $S_{\rho} \cap V$ links with $C$, then we may conclude that $b \geq \alpha>0$ is a critical value of $J_{\lambda}$ with nontrivial critical point.

LEMMA 3.2. - For all $\sigma \in \Sigma$,

$$
\sigma\left(B^{2}\right) \cap S_{\rho} \cap V \neq \emptyset
$$

Proof. - Assume the contrary: there exist $\sigma \in \Sigma$ such that $\sigma\left(B^{2}\right) \cap S_{\rho} \cap$ $V=\emptyset$. We define a family of maps,

$$
\begin{gathered}
F_{t}:\left(B_{\rho} \cap V\right) \times S^{1} \rightarrow H_{0}^{1}(\Omega) \\
F_{t}(z, \xi)=z-\sigma(t \xi), \quad \text { for } t \in[0,1], z \in B_{\rho} \cap V, \xi \in S^{1} .
\end{gathered}
$$

Note that (by assumption above), if $(z, \xi) \in \partial\left(\left(B_{\rho} \cap V\right) \times S^{1}\right)=$ $\left(S_{\rho} \cap V\right) \times S^{1}$, then $F_{t}(z, \xi) \neq 0$. Hence $\operatorname{deg}\left(F_{t},\left(B_{\rho} \cap V\right) \times S^{1}, 0\right)$ is 
a constant for all $t \in[0,1]$. By reparametrizing $D^{2}$ if necessary, we may assume that $\sigma(0) \notin B_{\rho} \cap V$. Hence, $F_{0}(z, \xi)=z-\sigma(0) \neq 0$ for all $z \in B_{\rho} \cap V$, and hence

$$
\operatorname{deg}\left(F_{t},\left(B_{\rho} \cap V\right) \times S^{1}, 0\right)=0 \quad \text { for all } t \in[0,1] .
$$

On the other hand, when $t=1, F_{1}(z, \xi)=z-\sigma(\xi)=z-\psi(\xi)$. By the construction of the curve $C, F_{1}\left(z, e^{i \theta}\right)=0$ has the unique solution $z=0$ and $\theta=0$. By the excision property of the degree,

$$
\operatorname{deg}\left(F_{1},\left(B_{\rho} \cap V\right) \times S^{1}, 0\right)=\operatorname{deg}\left(F_{1}\left(z, e^{i \theta}\right),\left(B_{\rho} \cap V\right) \times(-\varepsilon, \varepsilon), 0\right) .
$$

To calculate this degree, deform the $\operatorname{arc} \psi\left(e^{i \theta}\right), \theta \in(-\varepsilon, \varepsilon)$ to the straight segment, $\theta e_{1}$. Then, we have

$$
\begin{aligned}
& \operatorname{deg}\left(F_{1}\left(z, e^{i \theta}\right),\left(B_{\rho} \cap V\right) \times(-\varepsilon, \varepsilon), 0\right) \\
& \quad=\operatorname{deg}\left(z-\theta e_{1},\left(B_{\rho} \cap V\right) \times(-\varepsilon, \varepsilon), 0\right)=-1,
\end{aligned}
$$

a contradiction.

In conclusion, $S_{\rho} \cap V$ links with $C$, so by the linking theorem of Benci \& Rabinowitz [4], $b>0$ is a critical value of $J_{\lambda}$. This completes the proof of Theorem 3.1.

\section{APPENDIX}

First, we show by an example that, depending on the function $h$, the value $\lambda_{*}$ could be arbitrarily large. Without loss of generality, suppose that $B_{\varepsilon}(0) \subset \Omega$, for some $\varepsilon>0$. Define a sequence $\left\{h_{n}\right\}$ of smooth functions on $\mathbb{R}^{N}$ with $-1 \leq h(x) \leq 1$ and

$$
h_{n}(x)= \begin{cases}-1, & \text { when }|x| \leq \frac{1}{4} \frac{\varepsilon}{n}, \\ 0, & \text { when } \frac{1}{2} \frac{\varepsilon}{n} \leq|x| \leq \frac{3}{4} \frac{\varepsilon}{n}, \\ 1, & \text { when } \frac{\varepsilon}{n} \leq|x| .\end{cases}
$$

Clearly, $\int_{\Omega} h_{n}(x) e_{1}^{p} d x>0$ for all $n$ sufficiently large, and hence the corresponding values $\lambda_{*}=\lambda_{*}^{(n)}>\lambda_{1}(\Omega)$. Suppose $\lambda_{*}^{(n)} \leq M$ for all $n$. Each minimization problem is attained at some $v_{n} \in H_{0}^{1}(\Omega)$ with $\left\|\nabla v_{n}\right\|_{2}^{2}=\lambda_{*}^{(n)} \leq M,\left\|v_{n}\right\|_{2}=1$, and $\int_{\Omega} h_{n}\left|v_{n}\right|^{p} d x=0$. For some 
subsequence we have $v_{n} \rightarrow v_{0}$ in $H_{0}^{1}(\Omega)$ with $\left\|v_{0}\right\|_{2}=1$. Since $h_{n} \rightarrow 1$ in $L^{t}(\Omega)$ for all $t<\infty$ and $p<\frac{2 N}{N-2}$, we have $\int_{\Omega}\left|v_{0}\right|^{p} d x=0$, a contradiction.

We conclude with a proof of the Palais-Smale condition (Theorem 2.6 under the hypothesis $i$., which suffices for Theorems 1.2, 1.3, and 1.5. Note that (2.10) implies in addition,

$$
\begin{gathered}
F(u)-\quad=O\left(u^{2}\right) \\
F(u)-\frac{1}{p} f(u) u=O\left(u^{2}\right),
\end{gathered}
$$

as $|u| \rightarrow \infty$.

Let $\left\{u_{n}\right\}$ be a (PS) sequence,

$$
\begin{aligned}
J_{\lambda}\left(u_{n}\right) & =\int_{\Omega} \frac{1}{2}\left(\left|\nabla u_{n}\right|^{2}-\lambda u_{n}^{2}\right)-h(x) F\left(u_{n}\right) d x \leq C \\
J_{\lambda}^{\prime}(u) \varphi & =\int_{\Omega} \nabla u_{n} \cdot \nabla \varphi-\lambda u_{n} \varphi-h(x) f\left(u_{n}\right) \varphi d x \\
& =\int_{\Omega} \nabla z_{n} \cdot \nabla \varphi d x
\end{aligned}
$$

where $z_{n} \rightarrow 0$ in $H_{0}^{1}(\Omega)$ and $\varphi \in H_{0}^{1}(\Omega)$ is any fixed function. First, we claim $\left\|u_{n}\right\|_{2} \leq C$. Suppose the contrary, and set $v_{n}=u_{n} /\left\|u_{n}\right\|_{2}$. From (4.3), (4.4), and hypothesis (4.2) we have,

$$
\begin{aligned}
\left(\frac{1}{2}-\frac{1}{p}\right)\left(\left\|\nabla v_{n}\right\|_{2}^{2}-\lambda\right) \leq & \frac{1}{\left\|u_{n}\right\|_{2}^{2}} \int_{\Omega} h(x)\left[F\left(u_{n}\right)-\frac{1}{p} f\left(u_{n}\right) u_{n}\right] d x \\
& +o(1)\left\|\nabla v_{n}\right\|_{2} \\
= & O(1)+o(1)\left\|\nabla v_{n}\right\|_{2}
\end{aligned}
$$

Hence, $v_{n}$ is bounded in $H_{0}^{1}(\Omega)$ and a subsequence (which we still denote by $v_{n}$ ) converges weakly $v_{n} \rightarrow v_{0}$ in $H_{0}^{1}(\Omega)$. From (4.4) and (2.10) and for each fixed $\varphi \in H_{0}^{1}(\Omega)$ we obtain:

$$
\begin{aligned}
\left\|u_{n}\right\|_{2}^{p-2} \int_{\Omega} h(x)\left|v_{n}\right|^{p-2} v_{n} \varphi d x & =\frac{1}{\left\|u_{n}\right\|} \int_{\Omega} h(x)\left|u_{n}\right|^{p-2} u_{n} \varphi d x \\
& =\frac{1}{\left\|u_{n}\right\|} \int_{\Omega} h(x) f\left(u_{n}\right) \varphi d x+O(1) \\
& =\int_{\Omega}\left[\nabla v_{n} \cdot \nabla \varphi-\lambda v_{n} \varphi\right] d x+O(1) \\
& =\int_{\Omega}\left[\nabla v_{0} \cdot \nabla \varphi-\lambda v_{0} \varphi\right] d x+O(1)=O(1) .
\end{aligned}
$$


In particular

$$
\int_{\Omega} h(x)\left|v_{0}\right|^{p-2} v_{0} \varphi d x=0
$$

for all $\varphi \in H_{0}^{1}(\Omega)$. Hence, $v_{0} \in H_{D}^{1}\left(\Omega_{0}\right)$ and $\left\|v_{0}\right\|_{2}=1$, so $v_{0} \not \equiv 0$. Finally, taking $\varphi \in H_{D}^{1}\left(\Omega_{0}\right)$ we apply (4.4) again to obtain:

$$
\int_{\Omega_{0}} \nabla v_{0} \cdot \nabla \varphi-\lambda v_{0} \varphi d x=0
$$

which yields a contradiction unless $\lambda \in \sigma\left(\Omega_{0}\right)$. We conclude that $\left\|u_{n}\right\|_{2} \leq C$.

Now, combining (4.3) and (4.4) and using (4.2) we have:

$$
\begin{aligned}
\left(\frac{1}{2}-\frac{1}{p}\right)\left(\left\|\nabla u_{n}\right\|_{2}^{2}-\lambda\left\|u_{n}\right\|_{2}^{2}\right) \leq c & +\int_{\Omega} h(x)\left[F\left(u_{n}\right)-\frac{1}{p} f\left(u_{n}\right) u_{n}\right] d x \\
& +o(1)\left\|\nabla u_{n}\right\|_{2} \\
\leq & c\left\|u_{n}\right\|_{2}^{2}+c+o(1)\left\|\nabla u_{n}\right\|_{2} .
\end{aligned}
$$

Therefore, $\left\|\nabla u_{n}\right\|_{2}$ is uniformly bounded, and the subcritical growth of $F$ ensures that there is a strongly convergent subsequence.

\section{REFERENCES}

[1] S. Alama and G. Tarantello, On semilinear elliptic equations with indefinite nonlinearities, Calc. of Var. and P. D. E., Vol. 1, 1993, pp. 439-475.

[2] S. Alama and G. TARANTELlo, On the solvability of a semilinear elliptic equation via an associated eigenvalue problem, to appear in Math. $Z$.

[3] S. Alama and G. Tarantello, Elliptic problems with nonlinearities indefinite in sign, preprint, 1994.

[4] V. BENCI and P. RABINOWITZ, Critical point theorems for indefinite functionals, Invent. Math., Vol. 52, 1979, pp. 241-273.

[5] H. BeRESTYCKI, I. CAPUZZO-DolCETTA and L. NiRENBERG, Problèmes elliptiques indéfinis et théorèmes de Liouville non linéaires, C. R. Acad. Sci. Paris, t. 317, Série I, 1993, pp. 945-950.

[6] H. Berestycki, I. CAPUZZo-DolcetTa and L. Nirenberg, Variational methods for indefinite superlinear homogeneous elliptic problems, preprint, May 1994.

[7] H. BERESTYCKI, I. CAPUZZO-DOlCETTA and L. NiRENBERG, Superlinear indefinite elliptic problems and nonlinear Liouville theorems, preprint, 1994.

[8] H. BreZis and L. Nirenberg, " $H^{1}$ versus $C^{1}$ minimizers", $C$. R. Acad. Sci. Paris, t. 317, Série I, 1993, pp. 465-572.

[9] K. C. ChANG, "Infinite Dimensional Morse Theory and Multiple Solution Problems", Birkhäuser: Boston, 1993.

[10] K. C. Chang, " $H^{1}$ versus $C^{1}$ isolated critical points", C. $R$. Acad. Sci. Paris, t. 319, Série I, 1994, pp. 441-446. 
[11] M. DEL PINO and P. FELMER, Multiple solutions for a semilinear elliptic equation, preprint, 1992.

[12] J. F. EsCOBAR and R. M. SCHOEN, Conformal metrics with prescribed scalar curvature, Invent. Math., Vol. 86, 1986, pp. 243-254.

[13] H. HofER, A Note on the Topological Degree at a Critical Point of Mountainpass-type, Proc. Am. Math. Soc., Vol. 90, 1984, pp. 309-315.

[14] J. KAZDAN and F. WARNER, Scalar curvature and the conformal deformation of Riemannian structure, J. Diff. Geom., Vol. 10, 1975, pp. 113-134.

[15] J. MAWHIN and M. Willem, "Critical Point Theory and Hamiltonian Systems," Springer: New York, 1989.

[16] T. OUYANG, On the positive solutions of semilinear elliptic equations $\Delta u+\lambda u+h u^{p}=0$ on compact manifolds, Part II, Indiana Univ. Math. J., Vol. 40, 1992, pp. 1083-1140.

[17] P. Rabinowitz, "Minimax Methods in Critical Point Theory with Applications to Differential Equations," CBMS-NSF, Vol. 65, American Math. Soc.: Providence, 1986.

[18] M. STRUWE, "Variational Methods", Springer-Verlag: Berlin, 1990.

[19] H. Tehrani, Ph.D. thesis, New York Univ., 1994.

[20] Z. Q. WANG, On a superlinear elliptic equation, Ann. Inst. H. Poincaré-Analyse Non lin., Vol. 8, 1991, pp. 43-38.

(Manuscript received April 25, 1994;

Revised version received March 23, 1995;

Final version, March 1995.)

Vol. 13, $\mathrm{n}^{\circ} 1-1996$ 